\title{
Designing Automatic Meter Reading System Using Open Source Hardware and Software
}

\author{
Dragan Mlakić ${ }^{1}$, Srete Nikolovski ${ }^{2}$, Emir Alibašić ${ }^{3}$ \\ ${ }^{1}$ Distribution Company, Novi Travnik, Electric Power Company HZ-HB Inc. Mostar, Bosnia and Herzegovina \\ ${ }^{2}$ Power Engineering Department, Faculty of Electrical Engineering Computing and Information Technology, \\ Osijek, Croatia \\ ${ }^{3}$ ZZO Tuzla Kanton, Tuzla, Bosnia and Herzegovina
}

\begin{tabular}{l}
\hline Article Info \\
\hline Article history: \\
Received Apr 18, 2017 \\
Revised Jul 9, 2017 \\
Accepted Aug 8, 2017 \\
\hline Keyword: \\
Java \\
Electric energy measurement \\
Automatic Metering Reading \\
Arduino \\
Open source hardware \\
Linux application \\
Mobility
\end{tabular}

\begin{abstract}
The importance of quality of the measured values is very dependent on the device that measures these values: the size of the sample, the time of measurement, periods of measurement, the mobility and the robustness of the device, etc. Contemporary devices intended for the measurement of physical quantities that are on the market vary in price, as well as and the quality of the measured values. The rule "the more expensive the better" is not necessarily always a rule that is valid because it all depends on the characteristics and capabilities of the device, and the customer's needs. In this paper, a device based on "Open Source" components of hardware and software will be presented. Device was used to measure voltages and currents on low voltage networks, on which a virtually unlimited number of sensors can be added, and the device is assembled of components available on electronic components Internet.
\end{abstract}

Copyright $\odot 2017$ Institute of Advanced Engineering and Science. All rights reserved.

\section{Corresponding Author:}

Srete Nikolovski,

Departement of Power Engineering,

Faculty of Electrical Engineering Computing and Information Technology, Osijek,

Josip Juraj Strossmyer University of Osijek,

K. Trpimira 2B, 31000 Osijek, Croatia.

Email: srete.nikolovski@etfos.hr

\section{INTRODUCTION}

Automatic meter reading of smart meters is in contemporary standards, when Smart Grid standards are goal to be reached, the most important components of the network in all areas of distribution are: electricity, water supply, telecommunications, gas and liquid fuels. For companies engaged in such operations, it is imperative to better measure the raw materials that are distributed to their consumers, while the consumers aim to get the best quality of the desired product. Technology has in recent years made exceptional progress and brought plenty of new solutions, to measure the distance by AMR (Automatic Metering Reading) devices. What becomes the new standard is the spectrum of values to be measured, not aggressive measures, but ergonomic and environmentally acceptable, as the smaller the sample, the easier the availability of such information to the end user, whether it is network operator or a customer of supplied product (electric energy, information, water, gas ...). All of this is the basis for energy production and better prediction of energy values, which eventually lead to a more efficient distribution, production and smaller losses of energy and materials.

A few years ago, people used to manually read meters and write the states on the records, and that data was used to calculate consumers' electricity [1],[2]; however, not only the electricity but also the consumption of water, gas or liquid fuel. Technology has progressed and on places where measuring electricity was physically inaccessible, "smart meters" find their use. Smart meters that worked on the "push" 
principle of reading were used, with a limited sample, and GSM communication system was used that transmitted data as a text message from meters to the server, where the data was stored for the calculation of energy and maintenance of the measuring point [3]-[5]. However, the price dictated investment, so that the cost of data transmission over GSM card was profitable only in exceptional situations and smart meters were by price reserved for investments of large companies that were able to rely on the principle of "turn key" [5], [6]. Although the smart meter readings were leading in the technology of measuring values, which were important for the analysis of energy, technology advances opened the market of "Open Source Hardware" (OSH) and "Open Source Software" (OSS) devices and tools [7]. In the last few months, these markets have sufficiently technologically advanced. For a minimum cost, a measuring site can be put together including primary equipment for energy measurement, the measuring transducers of signals in digital form, processing units that collect the required signals [8], communication equipment and communication protocols that carry data [9], the server on which the program for the analysis and /or storage of data runs [8],[9]. In previous works, the collection of data from electric power plants was carried out only from the signal, which was already ready for download, storage within the memory [7]. OSH [7],[8],[10] were used as well as the Arduino Mega and PIC18LF4620 / CC2430 / S3C2440 systems, and as software the various cheap or protected products were used [7],[10], which have the ability to connect smart devices in "the Cloud" as an idea IoT (" Internet of Things "). What is not completely done, but is mentioned in [8] is the usage of the WiFi network that is highly adaptable to user requirements and can be implemented almost everywhere.

The work covers issues of installation of custom electronics, which collects data to the server where the user makes the application for gathering, storing, managing and/or manipulating data. The aim of the work is to analyze the market, to take advantage of available resources that an average household has within reasonable economic categories, and compile a smart device for measuring all desired values. Data string of the electric power values collected via OSH are: voltages (U), currents (I), and power (P). Many engineers are not strangers to OSH and OSS components presented in this paper. What is new is a presented multidisciplinary solution of problems which are suitable for households, research teams, academic institutions, laboratories.

The paper is made in the following order: the second chapter presents OSH and OSS components of which the whole device of the measuring site is assembled, parts of the measuring site. The third section presents assembling of the meter with a focus on adjusting the components in order to be within the standards of smart meters. The fourth chapter is testing of the meter and analysis of data collected from the measuring point and comparison with smart meters that are on the market. The fifth chapter concludes the topic of the paper.

\section{METHODOLOGY}

Automatic system of meter reading that relies on a Wi-Fi communication protocol has already been suggested in previous works [5],[9]. In this system, AMR uses Wi-Fi communication protocol to send data to a remote server. Data transfer is carried out within a specified period as a packet of information that includes everything a user of AMR system requires. Time period, in which a data transfer is carried out, is defined in the hardware when the parameters are set by the user. Packages sent via Wi-Fi protocols are within the structure of HTML (HyperText Markup Language) 1.0, which is also a tool for presentation of selected data on the chosen device.

The user is able to see the presentation of data on any device that has the ability to connect to Wi-Fi communication bus bar (mobile devices, tablets, portable computers, etc.), and that recognizes the HTML 1.0 scripting language. Due to the features of Wi-Fi (IEEE 802.11) protocol, such as unlimited data transfer through communication bus bar, easy and quick set up of all network settings, low-cost implementation, wireless connectivity, are the reasons this protocol is selected for the communication platform of the proposed AMR system. AMR possibilities that are available through the Wi-Fi protocol are expandable simply by assembling a new AMR meter that would over a Wi-Fi bus or directly from the Ethernet cable connect to the Wi-Fi router within the first AMR meter, by which the data packet would expand to a new LAN IP address and become available to the user. The methodology and architecture of AMR communication system are shown in Figure 1.

Information about current and voltage values are collected with the help of primary equipment at power cables using Current Measurement Transformers (SMT). This information is converted to analog and digital signals through the secondary measurement equipment that is located within the system of smart meter. The data is processed within the Arduino Uno R3 plate and transfer to the Wi-Fi router over ENC28J60 network module. Router transmits data to a Wi-Fi bus bar. Any device that has a HTML 1.0 browser and is connected to a local Wi-Fi network of the above mentioned routers can see the data in real time. However, a server application that collects data, keeps them and deposits them on the hard drive of a 
computer is also on a computer that is connected to a Wi-Fi bus bar router. The application collects data from the LAN address of the local network and stores them in the .txt file that is suitable for subsequent processing.

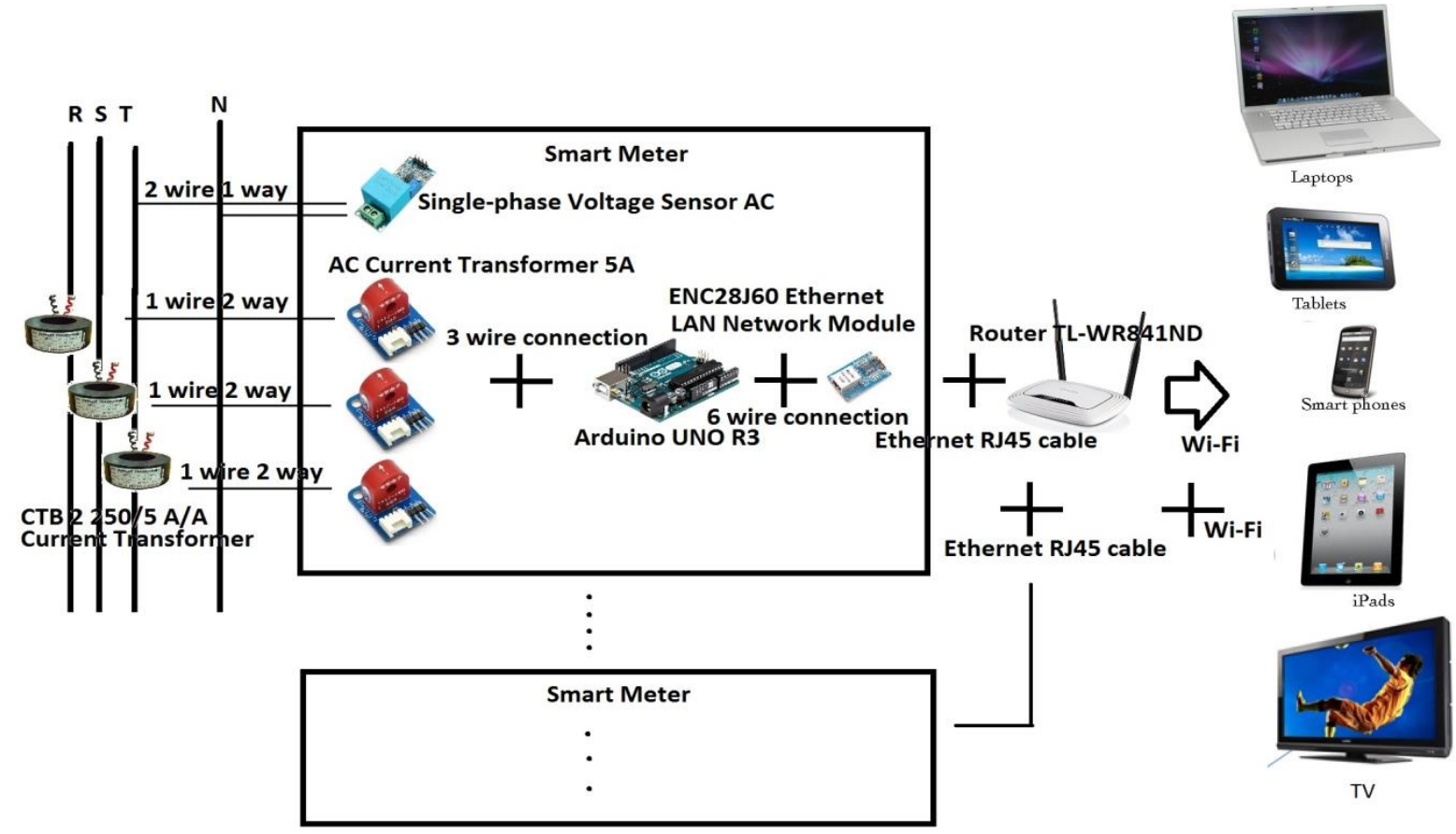

Figure 1. The architecture of the AMR system

\section{HARDWARE AND SOFTWARE ASSEMBLY}

All the components of which the AMR system is composed are available on the web stores of electronic goods. They are completely changeable and adaptable to the user. Because the market of OS equipment is unregulated, it is necessary to have additional knowledge when purchasing components, as not all components are equal in quality nor are all of them compatible with each other. Before purchasing, it is necessary to additionally inform oneself about the material that wants to be bought, figure out what is necessary, and what quality should be satisfied. Software is also the OS and is based mainly on the JAVA programming language. The programming tool for programming Arduino motherboard is available in all portals that deal with Arduino products.

\subsection{Hardware}

Firstly, the primary side of AMR system is explained. Current measurement transformer, which is used to measure the current with the power cable, is of the type ENTES ENT.B 75/5, 5VA, shown in Figure 2 .

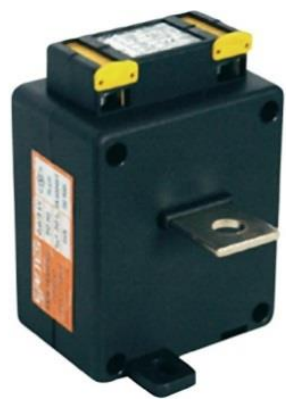

Figure 2. Current measurement transformer ENTES ENT.B 
Through the cable, it is connected to another current measurement transformer, which is exclusively designed for the Arduino board. Current transformer is based on TA12-100 current sensor that can measure current on the primary up to $5 \mathrm{~A}$, and reduce it to $5 \mathrm{~mA}$. The voltage is measured via voltage measuring transformers, which are directly linked to the Arduino board. Voltage measurement transformer is of a type ZMPT101B, shown in Figure 3.
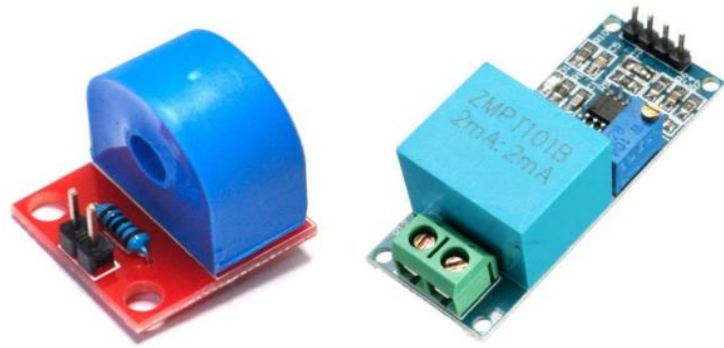

Figure 3. Current Measurement Transformer and Voltage measurement Transformer for the Arduino board

All primary equipment for measuring energy values of current (I) and voltage (U) have a relative error of 0.5 in relation to the measured value. Each measurement component as an output gives a sinusoidal shape of the analog signal, which is transmitted to the entrance of the Arduino board. All measuring sensors (I and U) are connected to the Arduino Uno R3 board that processes the signals. Signal processing involves the collection of signals from analog inputs on the board, calculation of the RMS value of the signal and the assignment of variables that are then suitable for sending the HTML 1.0. Figure 4 shows an Arduino Uno R3 board.

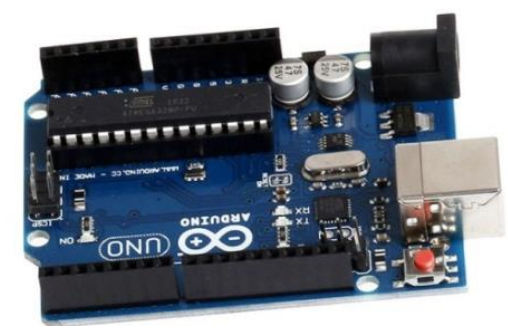

Figure 4. Arduino Uno R3 board

When the signals are encoded within the HTML code, they are forwarded then to a particular IP address with the help of ENC28J60 network module that this package of information through the TCP / IP protocol sends on ZYXEL 300 mbps router. Figure 5 shows the ENC28J60 network module for Arduino and ZYXEL router. Wi-Fi router is set to have an unlocked Wi-Fi network for the ease of managing experiment.
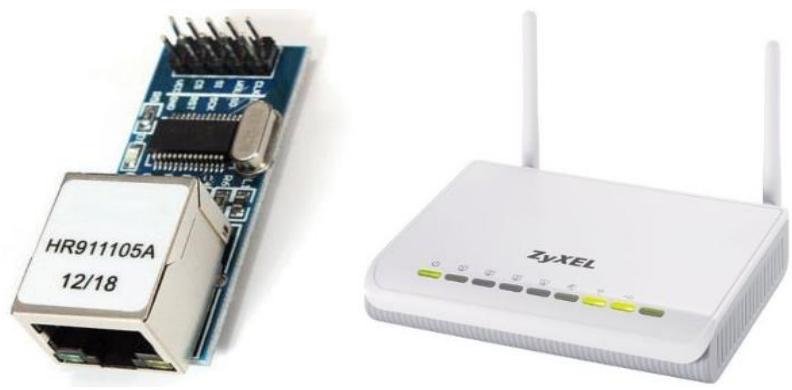

Figure 5. ENC28J60 network module for Arduino board and ZYXEL 300 mbps Wi-Fi router 
The computer on which the server of applications is carried out for collecting information is Fujitsu Lifebook S6410 with 2 GB of RAM. Regardless of the platform that is used in the role of the server, application will be performed with no difficulties. In the event of a problem with hardware, one can simply switch application to a different hardware and resume operation. Figure 7 shows the Fujitsu Lifebook S6410.

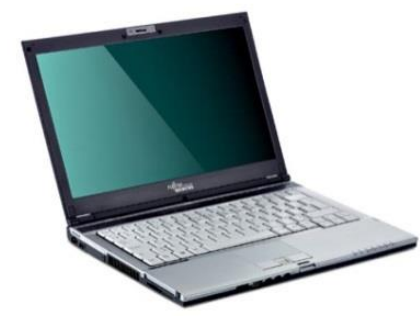

Figure 7. Fujitsu Lifebook S6410 in the role of a server for AMR

Finally, all mentioned is connected to a cardboard plate and fixed inside a suitcase made of hard plastic. An important feature of all these components related to Arduino is a requirement of a power supply via the Arduino Uno board, which is powered from a source of direct current $9 \mathrm{~V}$. All components are fastened with plastic laces, and all in the package is possible to transfer. Other components that were used are the board for joining electronic components, network cable with RJ45 interface, a thin $1.7 \mathrm{~mm} 2$ conductors for connecting components, adapters for supplying Arduino board and ZYXEL router from $9 \mathrm{~V}$ DC and $12 \mathrm{~V}$ DC, one extension cord for outdoor power. Figure 8 shows the entire HW system as closed and ready for transfer and open when it is in operation.

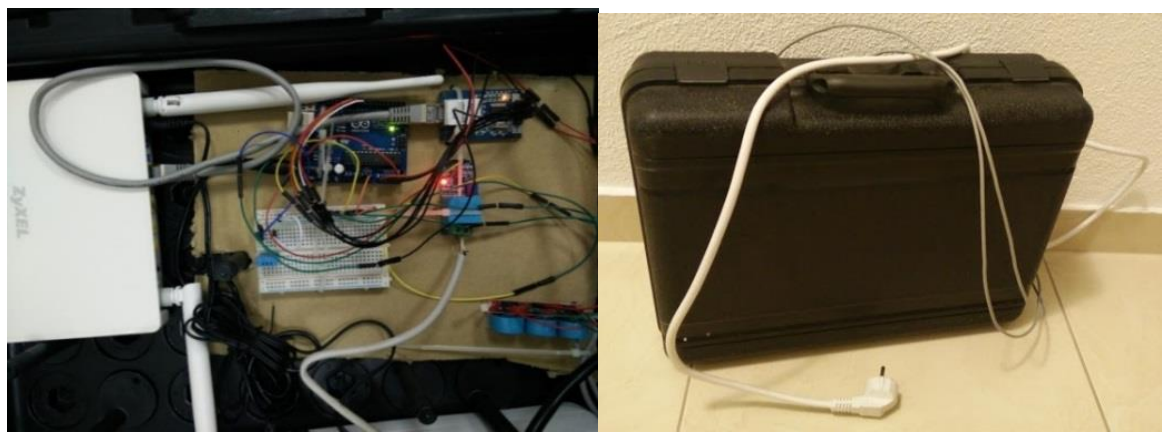

Figure 8. Left - opened bag with components in operation. Right - closed transfer suitcase

\subsection{Software}

The first OSS that is used is the program for programming Arduino UNO R3 board version 1.6.9. In this software tool, all operations related to the work of the Arduino components are defined in the:

- method of collecting sensor signals,

- addressing signals to a variable,

- calibration of values,

- inclusion of libraries that are essential for the operation of the sensor

- ways of sending data to the output that the user selects (in this case the IP address).

Figure 10 shows the programming interface for programming Arduino hardware. 


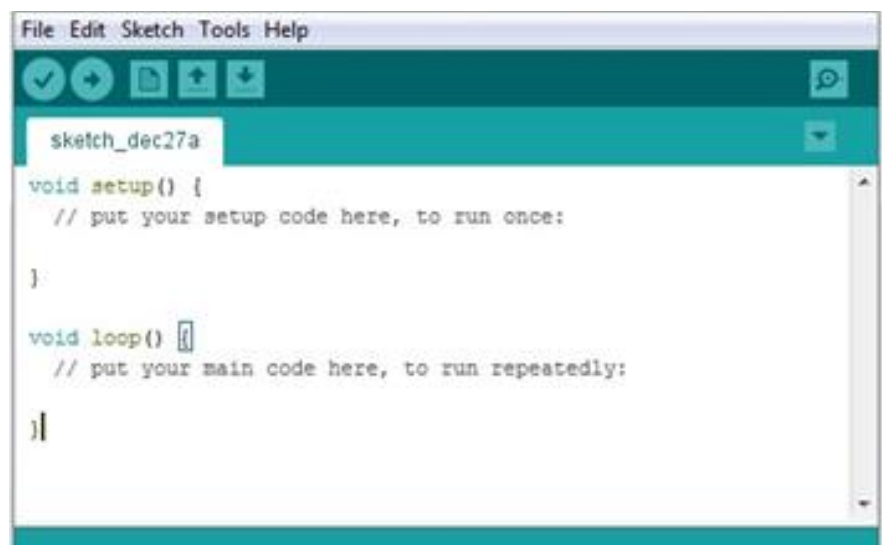

Figure 10. Application for programming Arduino UNO board

The following software that was used could have been any internet browser; in this case it was Google Chrome, which is available for free use. On the browser, the data is presented that are within the WiFi network and are updated in real time. The Figure 11 shows Chrome with data presented from the meter.

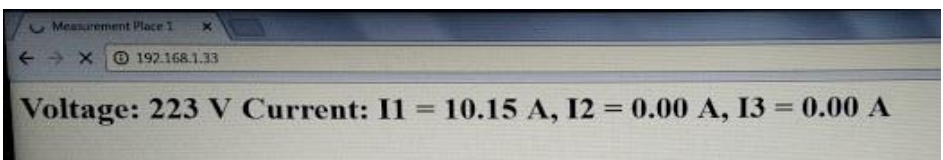

Figure 11. Google Chrome with data presentation from the meter

The operating system on which the server of application is carried out is Linux version of the Unix 7 32bit. The programming environment in which the application is written for meter reading in Java is the Eclipse Neon for Linux. Figure 12 shows the programming environment of Eclipse Neon for application development in Java.

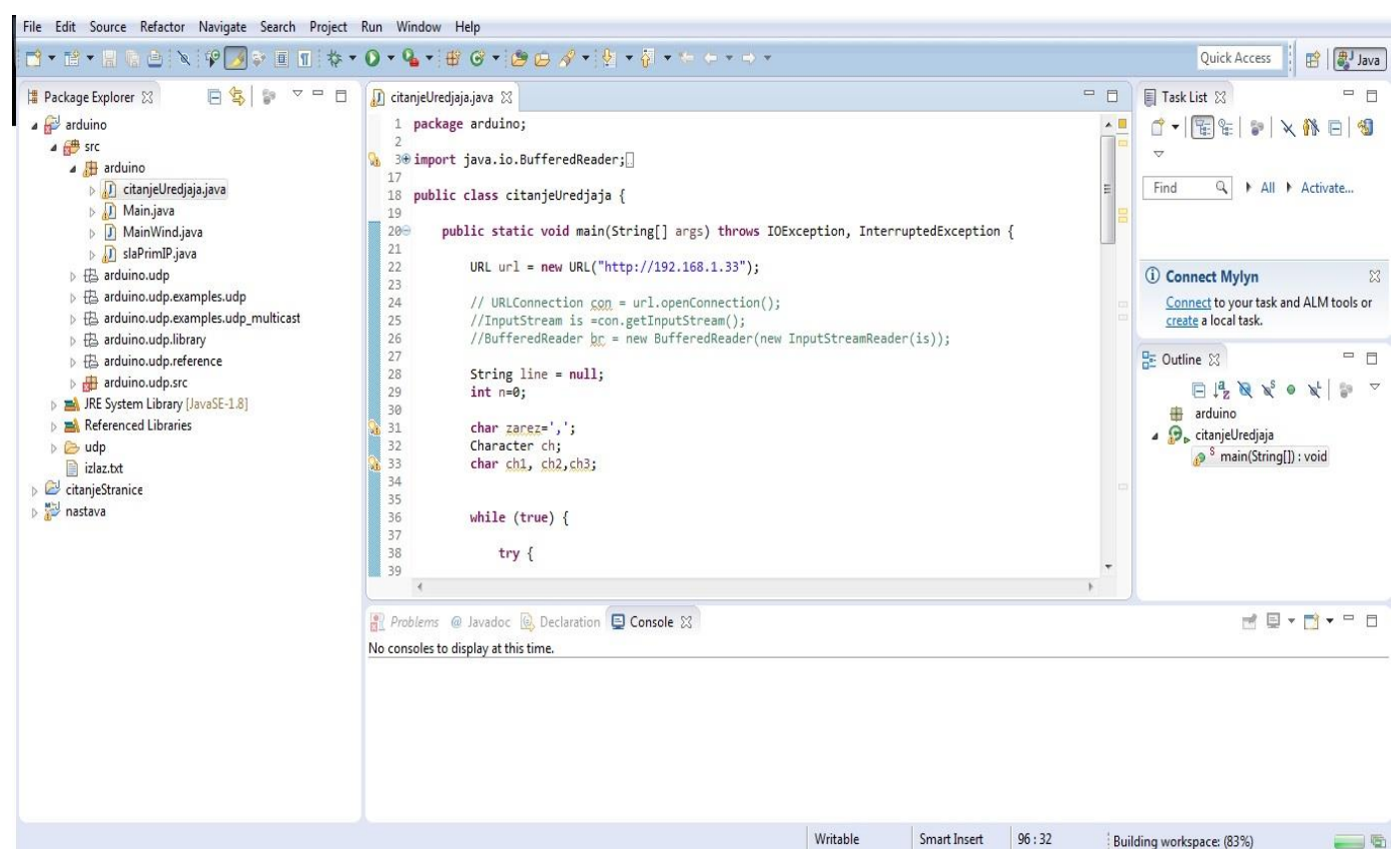

Figure 12. Eclipse Neon for development of JAVA application 
The server of application reads the IP address with all values that are presented on it and stores them in a * .txt file, that is later available for further data processing. Data from the IP address is read at time intervals of $200 \mathrm{~ms}$. The readings are far less common due to consumption of time required for calculating a signal from the sensor in RMS values that are transmitted to the IP address with the refresh time of 1s. Data transfer to a Wi-Fi bus bar takes about 1s. The speed processor used to run application has a delay of a few milliseconds. When all calculation is done, it yields a delay not less than 2 seconds. Usually, the delay may be from $2.5 \mathrm{~s}$ to $20 \mathrm{~s}$. Figure 13 shows the server of applications in the execution and txt file in which data is stored.
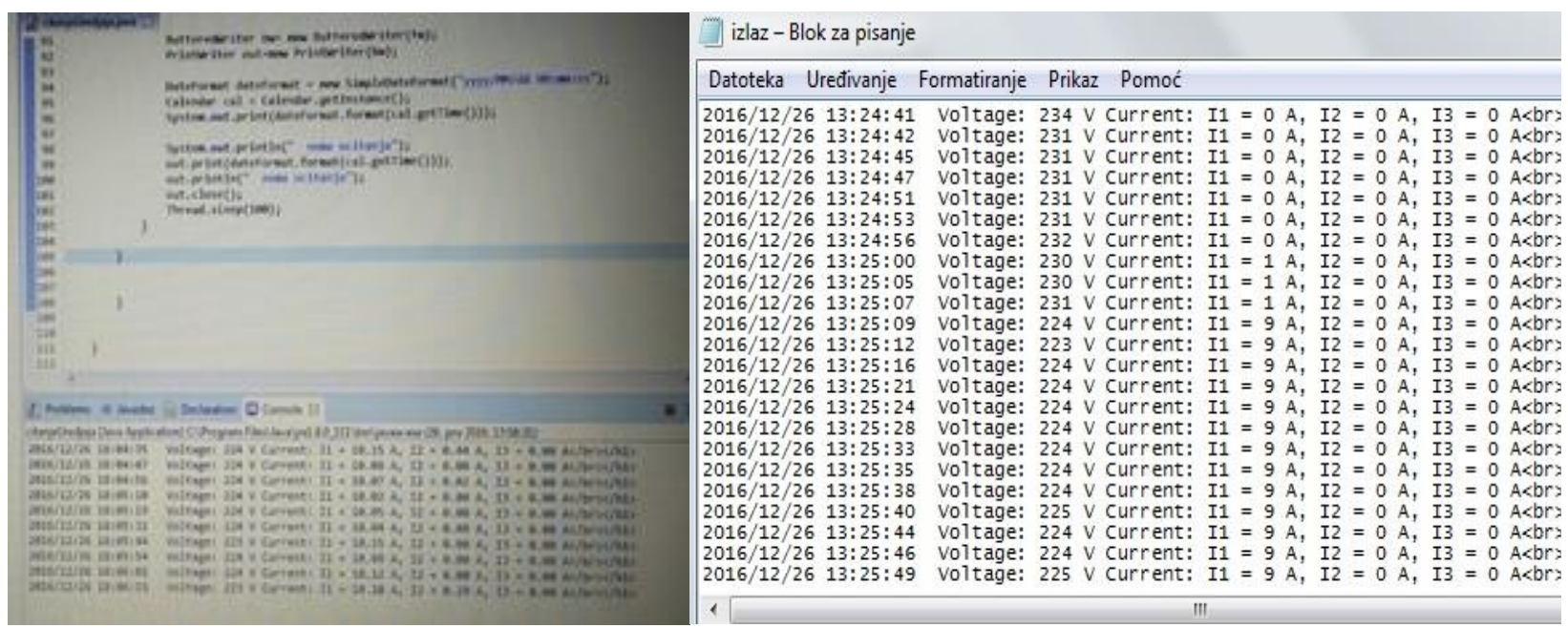

Figure 13. Left - server of applications when reading the ID address of the meter. Right - *.txt file with data stored from the application Figure 14

The flow of data through the architecture of AMR system with belonging formats is presented on

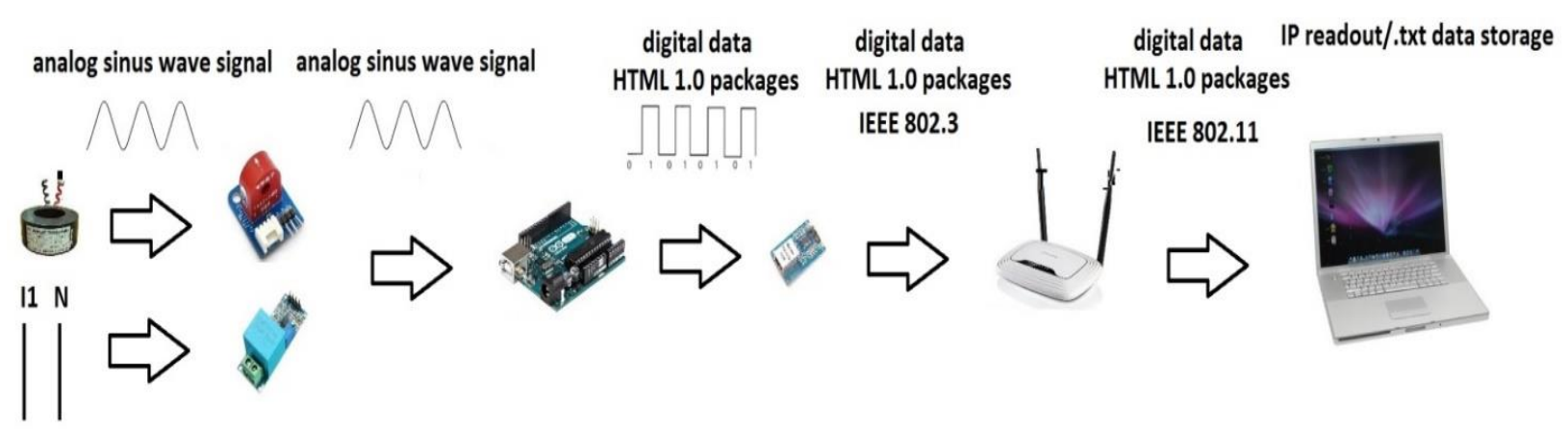

Figure 14. The flow of data through the architecture of AMR system

Extending the AMR system is done by attaching another set of measuring equipment with Arduino board to the ZYXEL router network cable. This involves one more IP address, which is again available for viewing in real time and recording with the application server.

\section{TESTING AND RESULTS}

Testing requires that each sensor is calibrated prior to data collection. Calibration is done every time loop for reading the signal from the sensor is started. The Arduino processor on which the program of smart meter runs does the job. For testing the system, a heater of $300 \mathrm{~W}$ was taken with two switches that can be 
independently switched on and off. For comparison, the timer used in regular distribution for the accounting measurement of electricity is set. The mentioned AMR system made of OSS and OSH is installed to measure from the same current transformer as the conventional mentioned meter. The tools that are still used are current clamp MEGGER DCM 300E for measuring current and MEGGER AVO300 for measuring voltage, a laptop for observing the measured values with a conventional meter. Figure 15 shows the entire practical and improvised "laboratory" in which the accuracy of the meter is measured.

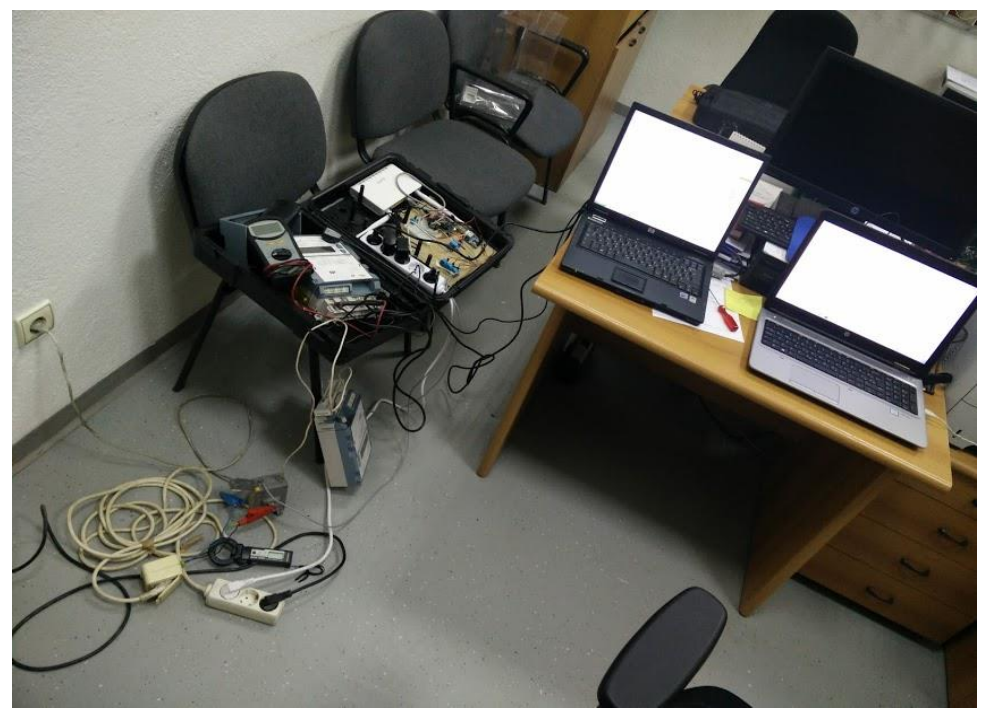

Figure 15. Set-up practical laboratory for measuring the accuracy of AMR system

Testing is done with the fan switched on the heater without two switches turned on, and then current and voltage with DCM 300E and AVO300 are measured and compared with the values indicated on the meter. After that, one switch is turned on and the same is done. Then, the process is carried out with both switches turned on. The measurement results are shown in Table 1. They show that from the tool the conventional meter accurately measures the voltage and current. Therefore, in the further analysis, the comparison between conventional AMR systems and OSS \& OSH AMR system was made

Table 1. Results of measurement values U and I

\begin{tabular}{cccc}
\hline Methods & $\begin{array}{c}\text { Devices } \\
\text { fan ON } \\
\text { switch1 OFF } \\
\text { switch 2 OFF }\end{array}$ & $\begin{array}{c}\text { Devices } \\
\text { fan ON } \\
\text { switch1 OFF } \\
\text { switch 2 OFF }\end{array}$ & $\begin{array}{c}\text { Devices } \\
\text { fan ON, } \\
\text { switch1 ON, } \\
\text { switch 2 ON }\end{array}$ \\
\hline AMR & $\mathrm{U}=233 \mathrm{~V}$ & $\mathrm{U}=228 \mathrm{~V}$ & $\mathrm{U}=223 \mathrm{~V}$ \\
OSS\&OSH & $\mathrm{I} 1=1,17 \mathrm{~A}$ & $\mathrm{I} 1=6,40 \mathrm{~A}$ & $\mathrm{I} 1=10,27 \mathrm{~A}$ \\
AMR & $\mathrm{U}=229,4 \mathrm{~V}$ & $\mathrm{U}=225 \mathrm{~V}$, & $\mathrm{U}=224 \mathrm{~V}$, \\
Conventional & $\mathrm{I} 1=1,06 \mathrm{~A}$ & $\mathrm{I} 1=6,48 \mathrm{~A}$ & $\mathrm{I} 1=10,60 \mathrm{~A}$ \\
\hline
\end{tabular}

Test of measuring energy consumption in a time of 60 minutes is made with both switches turned on and the fan on the heater. Both meters measured continuously and their results are recorded on computers on which the application is started. From conventional meter, the data is simply read each 15 minutes and recorded within the system for remote readings, while the designed AMR system documented data several times per minute and kept data in the * .txt file from which the consumption of energy is calculated after 60 minutes. The first 3 minutes is presented in Table 2, while the total energy consumption is displayed at the end of the table. 
Table 3. Sample of 3 minutes and sum of energy consumption for 60 minutes

\begin{tabular}{ccccc}
\hline Time & Voltage $(\mathrm{V})$ & Phase L1 & $\begin{array}{c}\text { Cuurents (A) } \\
\text { Phase L2 }\end{array}$ & Phase3L3 \\
\hline 2016/12/26 18:00:10 & 225 & 10.10 & 0.00 & 0.05 \\
2016/12/26 18:00:19 & 224 & 10.09 & 0.04 & 0.09 \\
2016/12/26 18:00:32 & 222 & 10.18 & 0.04 & 0.06 \\
2016/12/26 18:00:40. & 224 & 10.15 & 0.03 & 0.03 \\
2016/12/26 18:00:48. & 223 & 10.14 & 0.02 & 0.27 \\
2016/12/26 18:00:58 & 224 & 10.09 & 0.00 & 0.07 \\
2016/12/26 18:01:07 & 222 & 10.17 & 0.03 & 0.14 \\
2016/12/26 18:01:13 & 224 & 10.11 & 0.03 & 0.29 \\
2016/12/26 18:01:25 & 223 & 10.16 & 0.15 & 0.30 \\
2016/12/26 18:01:33 & 224 & 10.16 & 0.03 & 0.22 \\
2016/12/26 18:01:43 & 224 & 10.13 & 0.17 & 0.17 \\
2016/12/26 18:01:55 & 223 & 10.18 & 0.02 & 0.20 \\
2016/12/26 18:02:07 & 223 & 10.09 & 0.26 & 0.42 \\
2016/12/26 18:02:15 & 224 & 10.17 & 0.09 & 0.41 \\
2016/12/26 18:02:24 & 223 & 10.05 & 0.08 & 0.42 \\
2016/12/26 18:02:37 & 223 & 10.12 & 0.02 & 0.19 \\
2016/12/26 18:02:53 & 224 & 10.21 & 0.02 & 0.12 \\
2016/12/26 18:03:10 & 224 & 10.16 & 0.08 & 0.25 \\
$\ldots \ldots \ldots \ldots \ldots \ldots \ldots \ldots .$. & AMR OSS\&OSH & $2,25 \mathrm{kWh}$ & & Error \\
Sum of energy on 60 min & AMR Conventional & $2,37 \mathrm{kWh}$ & & $5,33 \%$ \\
\hline
\end{tabular}

\section{CONCLUSION}

This paper proved that it is possible to assemble AMR system with smart meter, taking into account the standards that are expected of such a system. The price of such system is with all components in a number of ways below the economic cost of registered branded systems that are available in the market. As can be seen from the results of testing OSS \& OSH, AMR system is by the characteristics under modern AMR systems. Due to its capabilities, the designed system has almost the same performances as modern systems. With a small database obtained (in this case * .txt files), it is possible to present and process data in any way that the user desires. This work has practically proved that the smart meters that can fit within the AMR system, are possible with the available material that can be cheaply purchased through the online store. The next step is to add additional sensors within the AMR system (heat, humidity, lighting, etc.), improving sampling measured values, expansion of data packages, which are placed in the server, improving the user interface (GUI) of application server, extending the capabilities of the database. From the current point, the user only determines the limit because the options are vast.

\section{ACKNOWLEDGEMENTS}

We would like to thank the Faculty of Electrical Engineering Computing and Information Technology, Osijek, for financial support to the authors in the PhD project program.

\section{REFERENCES}

[1] V. R. Gogineni, et al., "Real Time Domestic Power Consumption Monitoring using Wireless Sensor Networks," IJECE, vol/issue: 5(4), pp. 685 694, 2015.

[2] S. Singh and I. Chana, "Advance Billing and Metering Architecture for Infrastructure as a Service," International Journal of Cloud Computing and Services Science (IJ-CLOSER), vol/issue: 2(2), pp. 123-133, 2013.

[3] N. Wu, et al., "Design of the Remote Wireless Meter Reading System Based on GPRS," TELKOMNIKA, vol/issue: 11(11), pp. 6358 6366, 2013.

[4] A. Ahmed, et al., "Automatic Electric Meter Reading System: A Cost-Feasible Alternative Approach In Meter Reading For Bangladesh Perspective Using Low-Cost Digital Wattmeter and Wimax Technology,” International J. Eng. Tech, vol/issue: 8(3), pp. 800-807, 2011.

[5] M. I. Afridi, et al., "GSM Based Smart Distribution System," International Journal of Electrical and Computer Engineering (IJECE), vol/issue: 2(5), pp. 589 596, 2012.

[6] H. Shahinzadeh and A. H. Khosroshahi, "Implementation of Smart Metering Systems: Challenges and Solutions," TELKOMNIKA Indonesian Journal of Electrical Engineering, vol/issue: 12(7), pp. 5104-5109, 2014.

[7] N. A. M. Tahir, et al., "Design Automatic Meter Reading (AMR) Data Logger with Xbee," International Journal of Simulation: Systems, Science \& Technology, vol/issue: 13(1), pp. 67-73, 2012.

[8] S. Dessai, et al., "Design and Development of Low Cost Navigation and Security System for Indian Fisherman Using Adrino Nano Platform," International Journal of Reconfigurable and Embedded Systems, vol/issue: 4(1), pp. 28 41, 2015. 
[9] H. S. Huang, et al., "Research on the Wi-Fi Protocol Intelligent Automatic Meter Reading System," Advanced Materials Research, vol. 591-593, pp. 1523-1526, 2012.

[10] L. Q. Xi and L. Gang, "Design of remote automatic meter reading system based on ZigBee and GPRS," Proceedings of the Third International Symposium on Computer Science and Computational Technology (ISCSCT '10), 2010.

\section{BIOGRAPHIES OF AUTHORS}

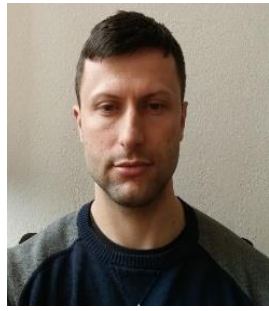

Dragan Mlakić, MSc, (IEEE M 2015) was born in Travnik on June 15, 1981. He obtained his BSc degree (2007) and MSc degree (2013), in electrical engineering at Faculty of Electrical Engineering, University of Sarajevo, Bosnia and Hercegovina. Presently he works as Engineer in Electric power company HZ-HB Inc. Mostar, Novi Travnik, Bosnia and Herzegovina and as Assistant Professor at the Software Engineering Department at Faculty of Information Technology, University Vitez, Bosnia and Herzegovina. His main interests are artificial intelligence, expert systems, renewable energy sources, energy quality, energy losses, system modeling, smart grid.

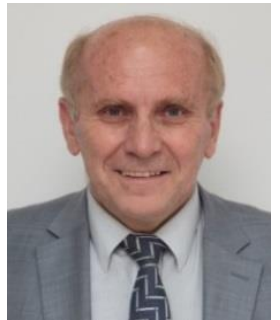

Srete Nikolovski, PhD.El.Eng. (IEEE M'1995, SM'2005) was born in Belgrade on October 1, 1954. He obtained his BSc degree (1978) and MSc degree (1989), in electrical engineering at the Faculty of Electrical Engineering, University of Belgrade and his PhD degree from the Faculty of Electrical and Computing Engineering, University of Zagreb, Croatia in 1993. Currently he is a Full Professor at Power Engineering Department at Faculty of Electrical Engineering, J.J. Strossmayer University in Osijek, Croatia. His main interests are power system protection, power system modeling, simulation and reliability. He has published 180 technical papers in journals and international conferences. He is a Senior Member of IEEE Reliability Society, PES Society and the member of Croatian National Committee of CIGRE.

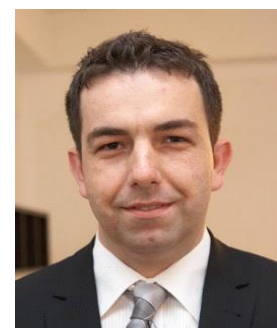

Emir Alibašić, Dipl.ing. electrical engineer, was born in Tuzla on March 12, 1982. He obtained his BCs degree (2007) in Tuzla in field of Electrical engineering at the Faculty of Electrical Engineering, University of Tuzla. Currently he is a $\mathrm{PhD}$ student at Faculty of Electrical Engineering, J.J. Strossmayer, University of Osijek, Croatia. His main interests are power transmission lines and optimization of electrical energy transmission. He was employed at UMEL-DALEKOVODMONTAZA Tuzla on the position of design and conduct of business in the electricity objects up to $400 \mathrm{kV}$ and owner INFORM Agency for the development of software applications. Currently he works as ITC manager in Tuzla county. 